\title{
Long-Term Effectiveness of Natalizumab in Patients with Relapsing-Remitting Multiple Sclerosis Treated in the Routine Care in Greece: Results from the Multicenter, Observational 5-Year Prospective Study 'TOPICS Greece'
}

\author{
Dardiotis Efthimios ${ }^{1} \cdot$ Karachalios Georgios $^{2} \cdot$ Alexopoulou Antonia $^{2} \cdot$ Gourgioti Rania $^{2}(D)$ \\ Evangelopoulos Maria-Eleutheria ${ }^{3}$. TOPICS Study Group
}

Accepted: 1 August 2021 / Published online: 24 August 2021

(c) The Author(s) 2021

\begin{abstract}
Background and Objectives For chronic diseases like multiple sclerosis (MS), real-world evidence on long-term treatment outcomes is essential. The study aimed to provide long-term data on the safety and effectiveness of natalizumab in patients with relapsing-remitting MS (RRMS) treated in a routine care setting in Greece.

Methods TOPICS Greece was a multicenter, single-country, prospective 5-year observational study.

Results Between 19-Apr-2012 and 18-Dec-2014, 304 eligible adults [females: 63.2\%; median age at natalizumab initiation: 38.0 years; median disease duration: 6.2 years; median Expanded Disability Status Scale (EDSS) score at baseline: 3.5$]$ were enrolled in the study by 20 hospital-based neurologists. The 1-year annualized relapse rate (ARR) before treatment initiation was 1.859, while the ARR during the first year of treatment was 0.131 , representing a significant $93 \%$ reduction $(p<0.001)$. The ARR over the median treatment period of 59.4 months was 0.109 . Patients with $\leq 1$ relapse in the prenatalizumab year $(46.1 \%)$ and those having received $\leq 1$ prior disease-modifying therapy (57.9\%) displayed significantly lower on-natalizumab ARR. The 1-, 2-, 3-, 4- and 5-year cumulative probabilities of EDSS progression were 3.2, 6.2, 9.7, 13.4 , and $17.4 \%$, respectively; the respective probabilities of EDSS disability improvement were 18.3, 25.1, 27.4, 28.0, and $30.1 \%$. Over a median safety data collection period of 48.7 months, $4.6 \%$ of the patients experienced $\geq 1$ serious adverse event, with infections (reported in 1.0\%) being the most common.

Conclusion In real-world settings in Greece, natalizumab displayed beneficial long-term effects on disease activity and disability progression consistent with previous studies with no new serious safety signals emerging.
\end{abstract}

\section{Introduction}

Multiple sclerosis (MS) is a chronic inflammatory central nervous system disease causing physical and cognitive disability $[1,2]$. The disease is more common among women

Gourgioti Rania

rgourgioti@genesispharma.com

1 Department of Neurology, Laboratory of Neurogenetics, University of Thessaly, University Hospital of Larissa, 41100 Larissa, Greece

2 CNS Department, Genesis Pharma SA, 270 Kifisias avenue, p.c. 15232, Athens, Greece

3 Demyelinating Diseases Unit, First Department of Neurology, School of Medicine, Eginition Hospital, National and Kapodistrian University of Athens, Athens, Greece

\section{Key Points}

In patients with relapsing-remitting multiple sclerosis (RRMS) treated with natalizumab in routine care setting in Greece, natalizumab yielded long-term benefits in terms of lowering the number of relapses and reducing disability progression.

The results of TOPICS Greece complement previous studies, indicating that natalizumab is a highly effective treatment for RRMS with an expected list of serious adverse events, thus, supporting its use, under the recommended risk minimization measures, for patients with highly active disease. 
and is usually diagnosed in young adulthood. Relapsingremitting MS (RRMS) is the most common type of MS, characterized by episodes of acute demyelination in the brain and the spinal cord (known as attacks or relapses), which last more than $24 \mathrm{~h}$ and result in the formation of lesions and the impairment of neuronal structure and function; these attacks are usually separated by at least one month of remission from the next episode. During remission, symptoms are less severe, though with progressing neurodegeneration residual disease becomes more pronounced.

Although the underlying pathogenetic mechanisms are not entirely understood, lymphocyte migration across the blood-brain barrier is recognized as a critical step in lesion formation in RRMS [3]. At the molecular level, this process is mediated by interactions between the $\alpha 4 \beta 1$ integrin on the lymphocyte surface and the vascular-cell adhesion molecule 1 on the vascular endothelial cell surface $[4,5]$. Interaction of $\alpha 4 \beta 1$ integrin with other molecules, such as fibronectin and osteopontin, is also implicated in modulation of leukocyte survival, priming, and activation, promoting inflammation $[6,7]$.

Natalizumab is a humanized recombinant monoclonal antibody approved for the treatment of RRMS [8]. The drug binds to the $\alpha 4$ subunit of integrins and blocks their binding to their endothelial receptors and other ligands, thereby inhibiting inflammation [9-11]. The safety and efficacy of natalizumab as a monotherapy for the treatment of RRMS was established in the Phase 3 randomized placebocontrolled clinical trial AFFIRM [12]. Over the course of two years, natalizumab reduced the annualized relapse rate (ARR) by $68 \%$, and the risk of sustained disability progression by $42 \%$, while it also reduced the number of new or enlarging brain lesions as visualized by magnetic resonance imaging (MRI).

For chronic diseases, such as MS, real-world data on long-term treatment outcomes are essential when evaluating a particular therapy. To this end, a number of real-world studies have been designed to assess effectiveness of natalizumab over longer observation periods than those commonly employed in clinical trials. In addition, these studies include a population with broad eligibility criteria that is more representative for the RRMS population receiving the drug under routine care conditions, compared to the strictly selected groups described in the clinical trial setting.

In light of the above, the TOPICS Greece 5-year prospective observational study was designed to assess the longterm safety and effectiveness of natalizumab in patients with RRMS treated in routine clinical care conditions in Greece. The present manuscript focuses on the effectiveness of natalizumab on disease activity and disability progression and examines baseline characteristics and short-term outcomes as prognostic indicators of disease activity.

\section{Methods}

\subsection{Study Design and Patient Population}

'TOPICS Greece' was a real-world, multicenter, prospective, observational study conducted in 20 hospital study sites across Greece. The study enrolled patients with RRMS who were therapy-naïve to natalizumab and to whom natalizumab (TYSABRI $^{\circledR}$, Biogen Netherlands B.V., FUJIFILM Diosynth Biotechnologies Denmark ApS) was initiated according to the locally approved label. The decision to initiate treatment with natalizumab, administered via intravenous infusion, had been taken prior to enrollment. Eligible patients had received $\leq 3$ natalizumab infusions before enrollment in the study, unless they were enrolled during the 6-month periods after approval of the original protocol and protocol amendment I or during the 3-month period after approval of protocol amendment II by the competent regulatory bodies, during which periods there was no restriction in the number of prior natalizumab infusions received (the purpose of these periods was to facilitate recruitment). Patients from Greece enrolled in the Tysabri Observation Program (TOP) study could be transferred to the TOPICS Greece database.

All patients provided informed consent for their participation in the study and were planned to be followed up for 5 years following informed consent signing. Only prospective safety data collection took place, i.e., adverse events (AEs) that had been experienced prior to informed consent signing were not recorded. Discontinuation of treatment with natalizumab was not considered a reason to terminate the patient's participation in the study. The study protocol and its amendments were approved by the competent national regulatory authority [National Organization for Medicines (EOF)] and the scientific committees of the participating hospitals and was conducted according to the ethical principles of the Declaration of Helsinki and all applicable national regulatory requirements. All participants provided written informed consent.

\subsection{Study Objectives and Endpoints}

The objectives of the study were to evaluate the long-term safety and effectiveness of natalizumab on disease activity and disability progression, as a single disease-modifying therapy (DMT) in patients with RRMM in a real-world clinical practice setting. The primary study objective was to assess the safety of natalizumab by determining the incidence of serious adverse events (SAEs). The effectiveness of therapy on disability progression was evaluated by estimating the proportion of patients whose Expanded Disability Status Scale (EDSS) score increased by at least 1.0 point and was sustained for 6 months, while disability improvement 
was defined as decrease from the EDSS reference value of at least one point sustained for at least 6 months. In addition, the proportions of patients reaching predefined EDSS milestones (4.0, 6.0 and 7.0) were assessed. Disease activity was examined by assessing the number of clinical relapses, the time to first relapse, the proportion of patients with relapse, and the ARR during the first and second year of treatment and throughout study participation. A clinical relapse was defined as new or recurrent neurological symptoms, not associated with fever, lasting for at least $24 \mathrm{~h}$, and followed by a period of 30 days of stability or improvement. New or recurrent neurological symptoms that occurred $<30$ days following the onset of a protocol-defined relapse were considered part of the same relapse. The study also aimed to examine baseline characteristics, as well as short-term (1-year) occurrence of relapse as prognostic indicators of disease activity. Furthermore, EDSS progression during the first 12 months after natalizumab initiation was to be evaluated as a prognostic indicator for disability progression over time, but due to the low number of EDSS progression events during the first year, this analysis was not performed.

In order to minimize bias stemming from betweenphysician differences of EDSS disability assessment, due the complexity and subjective nature of this assessment, physicians participating in TOPICS Greece were provided with an electronically available guide containing the definitions for a standardized, quantified neurological examination and assessment of Kurtzke's Functional Systems and EDSS, aiming to standardize and increase the quality of this assessment.

\subsection{Statistical Considerations}

No formal sample size estimation took place. Between 300 and 500 patients were planned to be enrolled in this study.

Statistical analysis was performed using SAS software (v9.4, SAS Institute, Cary, NC, USA). Data not following a normal distribution (as indicated by the Shapiro-Wilk test) are presented as median (interquartile range, IQR). Baseline was defined as the closest timepoint prior to or at natalizumab initiation. Differences in the EDSS from baseline to the post-baseline timepoints were assessed using the Wilcoxon signed-rank test. The Kaplan-Meier cumulative incidences of 6-month sustained EDSS disability progression and sustained EDSS disability improvement at 12, 24, 36, 48 and 60 months after baseline along with the 95\% confidence intervals (CIs) were estimated. Patients with no sustained EDSS disability progression event were censored at the time of the last EDSS assessment. Patients with no sustained EDSS disability improvement were censored at the time of the last EDSS assessment in case no sustained EDSS disability progression had occurred; otherwise, they were censored at the time of sustained EDSS disability progression. Six-month sustained EDSS disability progression or improvement was defined as an increase or decrease, respectively, from the reference value in the EDSS scores of at least one point sustained for at least 6 months, i.e., every EDSS score within a 6-month duration after the reference value met the examined criterion. The Kaplan-Meier cumulative incidences of first sustained EDSS score of 4, 6 , and 7 or greater at post-baseline timepoints along with the relevant $95 \%$ CIs were estimated among patients with a baseline EDSS assessment less than 4, 6 and 7, respectively. With the exception of censored data in the Kaplan-Meier analysis and partial dates, set to (1) the first day of the month for start dates with a missing start day, (2) the first month of the year for start dates with a missing month, (3) the last day of the month for end dates with a missing day, and (4) the last month of the year for end dates with a missing month, no imputation of missing data was applied.

For the estimation of the ARR throughout study participation, a Poisson model adjusted by scaled Pearson chi-square was constructed with the number of relapses for each patient since natalizumab onset as the response variable, and the natural log of time from natalizumab onset to last date on study as the offset term. Regarding the estimation of 1-year and 2-year ARRs after natalizumab initiation, the number of relapses in the period of interest were used as the response variable, and the natural log of time from natalizumab onset to last date on study was used as the offset term. For the identification of potential predictors of ARR throughout study participation, univariable and multivariable Poisson regression models were fitted. The models were adjusted by scaled Pearson chi-square in order to correct for overdispersion. The final multivariable model was derived through a stepwise procedure based on the minimization of Akaike's Information Criterion. Variables examined in the univariable analyses were included in the initial step of the stepwise procedure after excluding related variables. Factors with missing data $>20 \%$ were excluded from the multivariable model. All statistical tests were two-sided and were performed at a 0.05 significance level.

\section{Results}

\subsection{Safety}

During the prospective study observation period starting from the date of informed consent obtainment/enrollment in TOPICS until the last date on study [median (IQR): 48.7 (23.0-58.9) months], the SAE incidence rate was $4.6 \%$ (14/304), while the incidence rate of serious 'infections and infestations' was $1.0 \%$ (3/304), and those of serious opportunistic infections (all progressive multifocal 
leukoencephalopathy) and of malignancies were $0.7 \%$ (2/304). Safety results are presented in a separate manuscript [13].

\subsection{Patient Disposition and Baseline Characteristics}

Between 19-April-2012 and 18-December-2014, 335 patients were registered in the TOPICS Greece database, 304 of whom were eligible and have been analyzed. Of these, 241 patients were enrolled directly in TOPICS Greece, and 63 were transferred from TOP. Eligible patients had a median (IQR) age at natalizumab initiation (baseline) of 38.0 (29.0-45.5) years, and a median (IQR) disease duration of 6.2 (3.1-10.7) years. Baseline characteristics are presented in Table 1. The median (IQR) natalizumab treatment duration was 58.7 (30.7-78.0) months, and the median duration of study participation was 48.7 (23.0-58.9) months (with the last patient last visit performed on 29 October 2019). The median (IQR) time elapsed from natalizumab initiation to enrollment in TOPICS Greece was 0.7 months.

\subsection{Sustained EDSS Disability Progression and Improvement Rates During Study Participation and Attainment of EDSS Milestones}

Among patients with available paired assessments, the baseline EDSS score decreased at all examined post-baseline timepoints, with the difference being statistically significant at 12, 24, and 36 months (Fig. 1a). The 1-, 2-, 3-, 4and 5-year Kaplan-Meier estimated cumulative probability of 6-month sustained EDSS progression was 3.2\%, 6.2\%, $9.7 \%, 13.4 \%$, and $17.4 \%$ (Fig. 1b). The respective cumulative probabilities of 6-month sustained EDSS improvement were $18.3 \%, 25.1 \%, 27.4 \%, 28.0 \%$, and $30.1 \%$ (Fig. 1c).

The 1-, 2-, 3-, 4- and 5-year Kaplan-Meier estimated cumulative probabilities of reaching the EDSS milestone of $\geq 4.0$ were $3.1 \%, 5.7 \%, 8.8 \%, 12.5 \%$ and $15.2 \%$. The respective probabilities of reaching the EDSS milestone of $\geq 6.0$ were $1.7 \%, 3.0 \%, 4.5 \%, 5.1 \%$ and $5.7 \%$, while the 4- and 5-year probabilities of reaching the EDSS milestone

Table 1 Patient, disease and prior treatment characteristics

\begin{tabular}{|c|c|}
\hline Characteristic & Value \\
\hline Females $(N=304), n(\%)$ & $192(63.2)$ \\
\hline Age at baseline $(N=304)$, median $(\mathrm{IQR})$ & $38.0(29.0-45.5)$ \\
\hline$\leq 40$ years & $184(60.5)$ \\
\hline$>40$ years & $120(39.5)$ \\
\hline Disease duration at baseline $(N=299)$, median (IQR) & $6.2(3.1-10.7)$ \\
\hline$\leq 5$ years & $123(41.1)$ \\
\hline$>5$ years & $176(58.9)$ \\
\hline \multicolumn{2}{|l|}{ Relapses in the year prior to natalizumab initiation $(N=304), n(\%)$} \\
\hline$\leq 1$ & $140(46.1)$ \\
\hline$>1$ & $164(53.9)$ \\
\hline ARR in the year prior to natalizumab onset $(95 \% \mathrm{CI}),(N=304)$ & $1.859(1.708-2.023)$ \\
\hline ARR in the 2 years prior to natalizumab onset $(95 \% \mathrm{CI}),(N=304)$ & $1.355(1.261-1.455)$ \\
\hline \multicolumn{2}{|l|}{ Baseline EDSS score $(N=286)$, median (IQR) } \\
\hline$\leq 3$ points & $135(47.2)$ \\
\hline$>3$ points & $151(52.8)$ \\
\hline Positive anti-JCV serostatus at baseline $(N=72), n(\%)^{\text {a }}$ & $28(38.9)$ \\
\hline \multicolumn{2}{|l|}{ Prior DMTs $(N=304), n(\%)$} \\
\hline 0 & $37(12.2)$ \\
\hline$\geq 1$ & $267(87.8)$ \\
\hline$\geq 2$ & $128(42.1)$ \\
\hline Prior steroids/immunoglobulins $(N=304), n(\%)$ & $148(48.7)$ \\
\hline Prior antineoplastic/immunosuppressive therapy $(N=304), n(\%)$ & $20(6.6)$ \\
\hline Natalizumab treatment duration $(N=304)$, median (IQR), months & $58.7(30.7-78.0)$ \\
\hline Patients with permanent natalizumab treatment discontinuation $(N=304), n(\%)$ & $154(50.7)$ \\
\hline
\end{tabular}

$A R R$ annualized relapse rate, $C I$ confidence interval, $D M T$ disease-modifying therapy, EDSS Expanded Disability Status Scale, $I Q R$ interquartile range; $J C V$ John Cunningham virus, $N$ number of patients with available data

${ }^{a}$ Anti-JCV testing at baseline was not a prerequisite during the enrollment period of the study 
of $\geq 7.0$ were $0.5 \%$ and $2.3 \%$ (the 1-, 2-, and 3-year probabilities were zero).

\subsection{Annualized Relapse Rate and Relapse-Free Rate During the Study Observation Period}

A total of 173 relapses were experienced by 96 patients (31.6\%) during a median observation period of 59.4 (IQR: 37.4-79.8) months. A total of 97 relapses, experienced by 49 patients $(51.0 \%)$ required hospitalization, while 161 relapses, experienced by 88 patients $(91.7 \%)$ required steroid treatment. The ARR during the period between baseline and the end of study participation was 0.109 (95\% CI: 0.086, 0.138 ) relapses per patient-year. The 1- and 2-year ARR was 0.131 and 0.127 relapses/per patient year, respectively, corresponding to a $93.0 \%(p<0.001)$ and a $90.6 \%(p<$ 0.001 ) reduction from the one- and two-year periods prior to natalizumab initiation (Fig. 2a). The Kaplan-Meier estimated relapse-free rate at $12,24,36,48$, and at 60 months post-baseline was $92.7 \%, 89.4 \%, 82.4 \%, 78.1 \%, 70.7 \%$, and $68.2 \%$, respectively (Fig. $2 \mathrm{~b}$ ). The median time to first relapse could not be calculated since the survival curve does not drop to 0.5 or below (Fig. 2b), i.e., more than half of the patients remained relapse-free over the post-baseline observation period.

\subsection{Poisson Regression Analysis of Factors Associated with ARR}

The association of selected factors of interest with the ARR was evaluated by univariable and multivariable Poisson regression models. The factors examined are presented in Table 2. Based on the final multivariable model, in which the factors retained were sex, disease duration at baseline, number of relapses in the year prior to baseline and number of prior DMTs, the following associations were identified: (1) patients who experienced $\leq 1$ relapse in the year prior to baseline had an about 3 times lower ARR (0.058 vs 0.154 ) during the period between baseline and the end of study participation than those that experienced $>1$ relapses (ARR ratio: $0.356,95 \%$ CI: $0.221-0.572 ; p<0.001$ ), and (2) patients that had received $\leq 1$ prior DMTs had $50 \%$ lower ARR (0.082 vs 0.149$)$ than those that had received $\geq 2$ prior DMTs (ARR ratio $=0.535 ; p=0.004)$.

In a separate univariable model that examined the prognostic impact of the occurrence of relapse during the first year of natalizumab treatment with the ARR during the second year of treatment and beyond, the ARR during the second year and beyond of patients with a relapse during the first year was found to be 3.767 times higher than the ARR of those who did not experience a relapse during the first year of treatment $(p<0.001)$.

\section{Discussion}

TOPICS Greece demonstrates that in patients with RRMS treated in the routine clinical care in Greece, natalizumab demonstrates a safety profile consistent with that previously established, while it reduces MS disease activity as assessed by the ARR, and improves disability progression, as demonstrated by reductions in the EDSS score.

With respect to safety, in the present study natalizumab displayed a low SAE incidence rate (4.6\%), with no new safety signals emerging, and a low AE treatment discontinuation rate $(5.2 \%)$.

Regarding disability progression, in the present study, the EDSS score was significantly lower than baseline after the first, second and third year of treatment with natalizumab. Similarly, in the five-year interim analysis of TOP, the baseline EDSS score significantly decreased after one year of treatment and retained this value through the fourth year of observation [14]. Moreover, at two years post-baseline, the cumulative probability of 6-month sustained EDSS progression in TOPICS Greece was $6.2 \%$, lower than the $17 \%$ rate reported in AFFIRM and the $14.1 \%$ rate reported in the 2-year interim analysis of the STRIVE open-label, multicenter, Phase 4 study $[12,15]$. Notably, all patients in STRIVE were anti-JCV-antibody negative, with early RRMS, and baseline EDSS $\leq 4.0$ [14], restrictions which did not apply in TOPICS Greece. Furthermore, the 4- and 5-year Kaplan-Meier estimated cumulative probability of 6-month sustained EDSS progression in TOPICS Greece (13.4\% and $17.4 \%$ ) did not largely differ from the respective rates (14\% for both timepoints) reported in the 5-year interim analysis of TOP [13]. Similarly, the $25.1 \%$ 2-year cumulative probability of sustained EDSS improvement in TOPICS Greece was comparable to the $28.4 \%$ rate reported in STRIVE, while the 4- and 5-year rates (28\% and 30\%) were slightly higher than the $24 \%$ and $27 \%$ reported in the interim analysis of TOP, utilizing the same definitions both for disability progression and for improvement [13]. Moreover, in the present study, the 5-year Kaplan-Meier estimated cumulative probabilities of 6-month sustained EDSS milestones of $\geq 4.0, \geq 6.0$, and $\geq 7.0$, in patients with baseline EDSS <4.0, 6.0, and 7.0, respectively, were 15.2, 5.7, and $2.3 \%$, respectively. In a relevant analysis of TOP, the probabilities of 48-week confirmed transition from EDSS scores of $2.0-3.0$ to $\geq 4.0$, and $4.0-5.0$ to $\geq 6.0$ at Week 288 were 11.8 , and $9.5 \%$, respectively [16].

Natalizumab also had a positive effect on disease activity as assessed by ARR, which significantly improved from the years prior to treatment initiation and throughout the study observation period. Specifically, the ARR, decreased from 1.859 in the year prior to natalizumab initiation to 0.131 in the year post-treatment initiation corresponding 
Fig. 1 EDSS disability progression during study participation. Box-plots of the EDSS score at baseline and at the post-baseline timepoints (a). Numbers indicate median values; boxes extend from first to third quartile and whiskers from minimum to maximum score. Mean (SD) decrease from baseline derived from paired data along with $p$ values (Wilcoxon signed-rank test) are indicated; median decreases were not shown as they were all zero. Kaplan-Meier estimated cumulative probability of 6-month sustained EDSS progression (b). Estimated cumulative probabilities at the 12-, 24-, 36-, 48-, and 60-month timepoints are tabulated below the graph. Kaplan-Meier estimated cumulative probability of 6-month sustained EDSS improvement (c). Estimated cumulative probabilities at the 12-, 24-, 36-, 48-, and 60-month timepoints are tabulated below the graph. Analyses in $\mathbf{b}$ and $\mathbf{c}$ was performed among eligible patients with a baseline and at least one post-baseline EDSS assessment $(N=286)$. EDSS expanded disability status scale, $C I$ confidence interval, $N$ number of patients with available data a
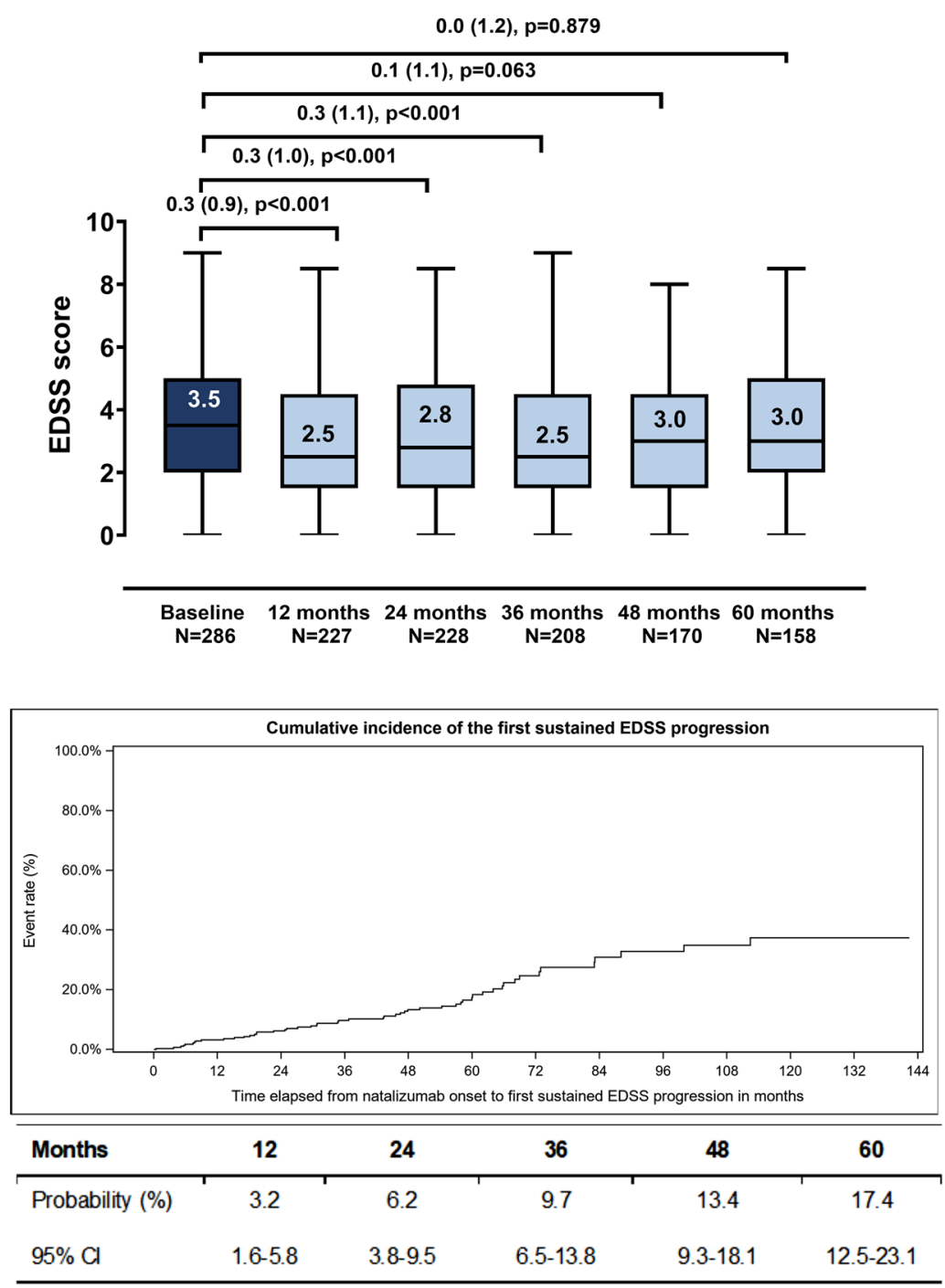

C

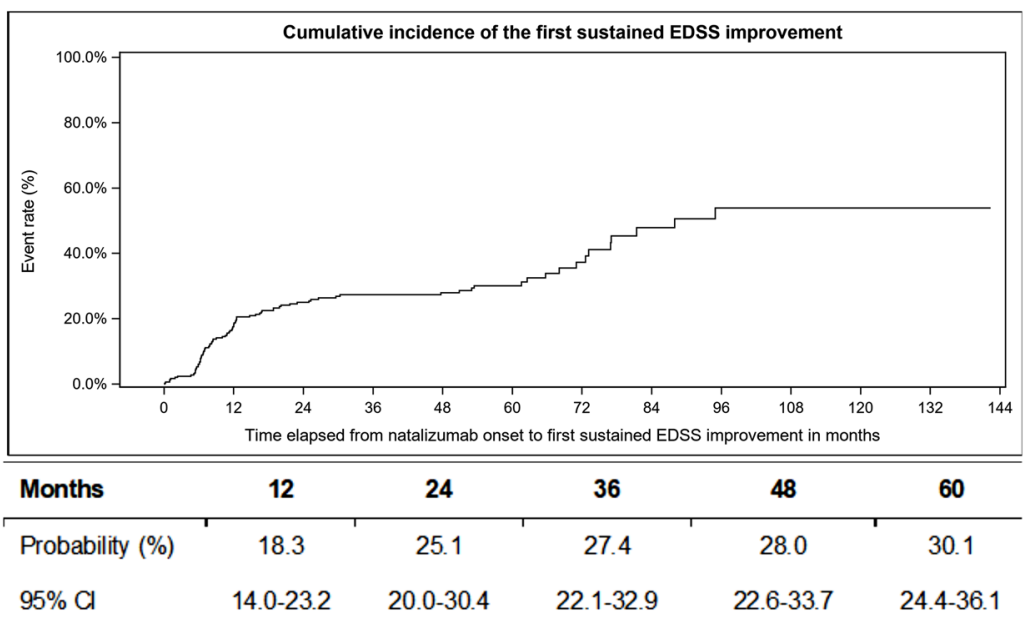

to a significant $93.0 \%$ reduction. The respective reductions according to the 5-year and 10-year analyses of TOP were $84.9 \%$ and $87.9 \%$, respectively $[13,17]$. Moreover, the ARR during the period between baseline and the end of study participation was 0.109 relapses per patient-year in TOPICS Greece, corresponding to a $94.1 \%$ reduction from the year 
Fig. 2 ARR and relapse-free rate during the study observation period. ARR during the indicated periods based on data from the overall eligible population (a). Kaplan-Meier estimated relapse-free rate during the study observation period (b). Estimated rates at the 12-, 24-, 36-, 48-, and 60-month timepoints are tabulated below the graph. ARR annualized relapse rate ratio, $C I$ confidence interval, $N$ number of patients with available data a

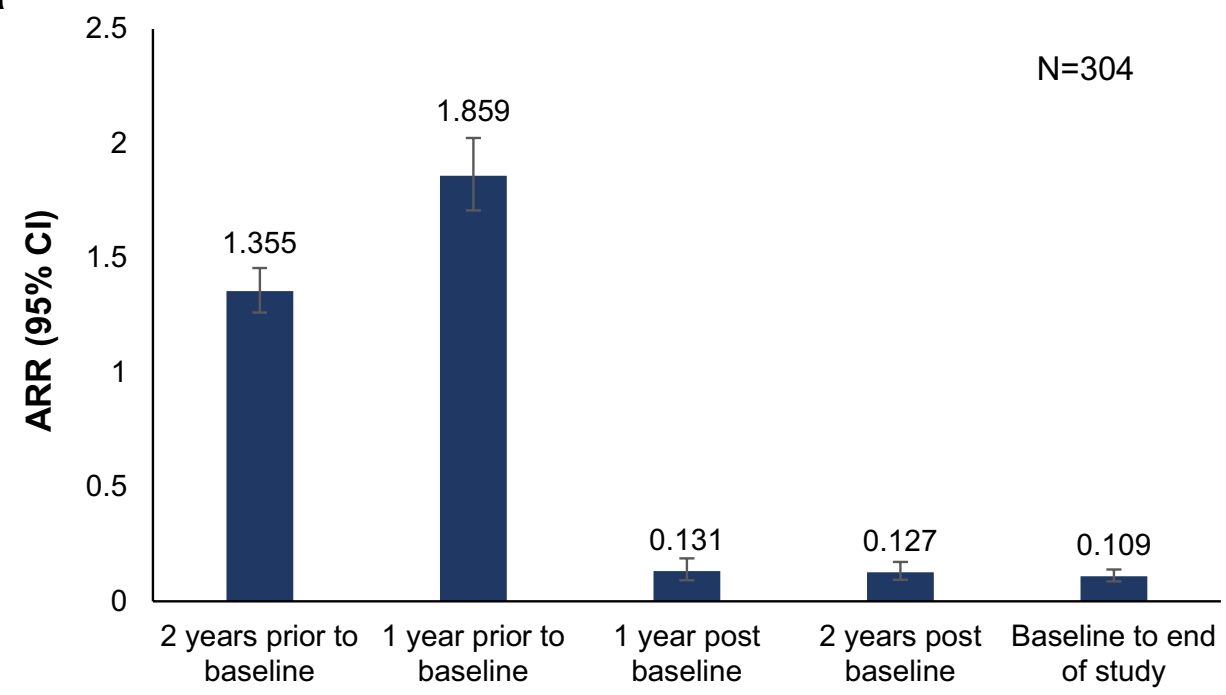

b

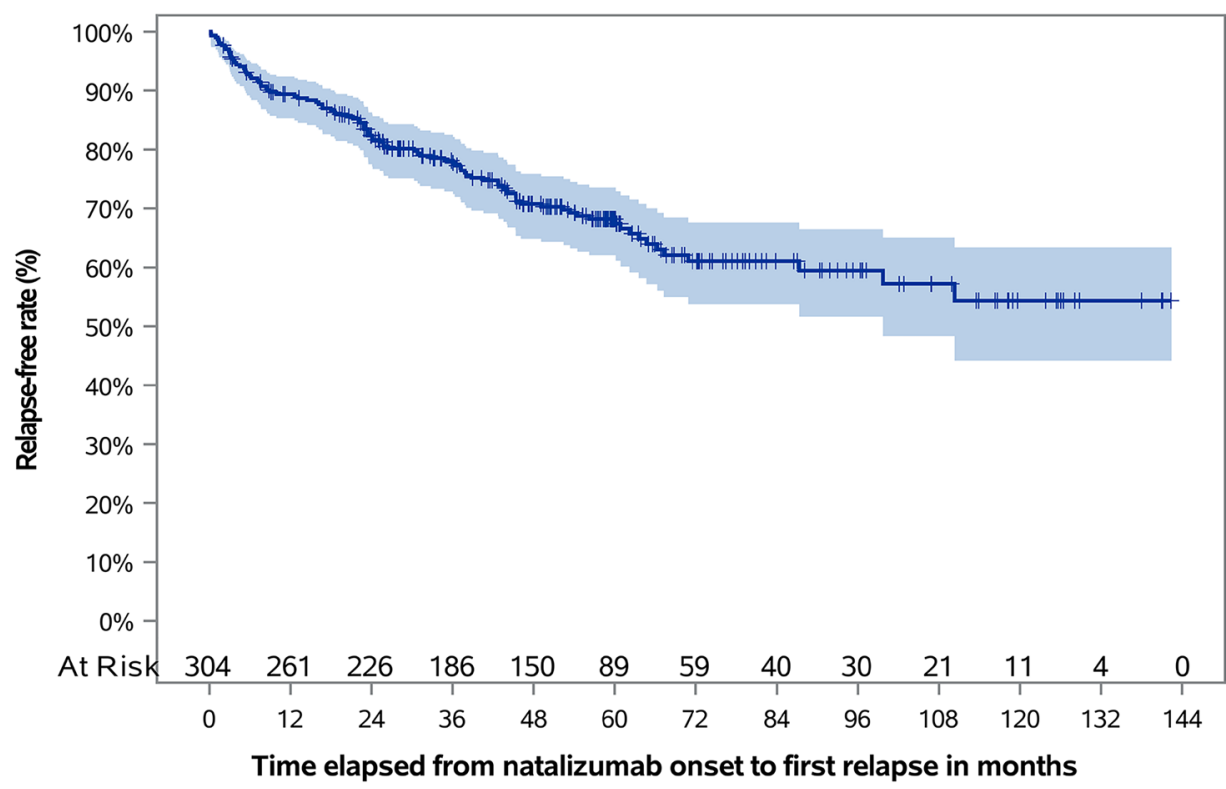

\begin{tabular}{lccccc}
\hline Months & 12 & 24 & 36 & 48 & \multicolumn{1}{c}{60} \\
\hline Rate (\%) & 89.4 & 82.4 & 78.1 & 70.7 & 68.2 \\
$95 \% \mathrm{Cl}$ & $85.3-92.4$ & $77.5-86.3$ & $72.9-82.5$ & $64.9-75.8$ & $62.1-73.5$ \\
\hline
\end{tabular}

prior to treatment initiation, while according to the 10-year data of TOP, the 10-year ARR was 0.15 corresponding to a $92.5 \%$ reduction from the year prior to baseline [16]. Similarly, the Kaplan-Meier estimated relapse-free rates in TOPICS Greece were higher than the rates reported for the respective timepoints in the 5-year interim analysis of TOP [13]. Other studies have also reported significant reduction in ARR after one and two years of natalizumab therapy $[14,18-20]$. For instance, in the BIONAT $_{2 Y}$ cohort, which included patients enrolled in BIONAT with at least 2 years of treatment, the ARR reduction was evaluated at $78.6 \%$ in the first year of treatment, with an additional $28.9 \%$ reduction in the second year; the respective rates in the subgroup of the BIONAT $_{2 Y}$ cohort with high disease activity at baseline $\left(\mathrm{BIONAT}_{\mathrm{HA} 2 \mathrm{Y}}\right.$ ) were $83.3 \%$ and $35.6 \%$ [17].

The above differences in the relapse-free rates and ARR among the different cohorts may at least in part be attributed to variation in the baseline characteristics. In particular, as 
Table 2 Poisson regression models of the association of selected factors with the ARR during the study observation period

\begin{tabular}{|c|c|c|c|}
\hline Parameter & Category vs reference & ARRR & $p$ value \\
\hline \multicolumn{4}{|l|}{ Univariable Poisson regression analysis } \\
\hline Sex & Male vs female & 0.861 & 0.557 \\
\hline Age at baseline (years) & $\leq 40$ vs $>40$ & 1.082 & 0.753 \\
\hline Disease duration at baseline (years) & $\leq 5$ vs $>5$ & 0.787 & 0.333 \\
\hline Number of relapses in the year prior to baseline & $\leq 1$ vs $>1$ & 0.377 & $<0.001$ \\
\hline Number of prior DMTs & $\leq 1$ vs $\geq 2$ & 0.546 & 0.008 \\
\hline Baseline EDSS & $\leq 3$ vs $>3$ & 0.900 & 0.677 \\
\hline \multicolumn{4}{|c|}{ Multivariable Poisson regression analysis; 299 observations } \\
\hline Sex & Male vs female & 0.846 & 0.463 \\
\hline Disease duration at baseline (years) & $\leq 5$ vs $>5$ & 0.826 & 0.388 \\
\hline Number of relapses in the year prior to baseline & $\leq 1$ vs $>1$ & 0.356 & $<0.001$ \\
\hline Number of prior DMTs & $\leq 1$ vs $\geq 2$ & 0.535 & 0.004 \\
\hline
\end{tabular}

$A R R R$ annualized relapse rate ratio, $D M T$ disease-modifying therapy, EDSS expanded disability status scale

derived from the univariable and multivariable analyses presented herein, and in agreement with other reports, a lower number of relapses in the year prior to natalizumab initiation and exposure to fewer prior DMTs, are associated with lower post-baseline ARR [13, 16]. Pre-baseline ARR has also been identified as a predictor for maintaining no evidence of progression or active disease over a longer time under treatment with natalizumab [21], while in the present study it was shown that occurrence of a relapse during the first year of treatment is a negative prognostic factor of relapse occurrence in the second and beyond year of treatment. Such analyses are particularly useful in helping characterize the patient profile that could derive the maximum treatment benefit from a specific therapy. In addition to such studies, evidence generated from meta-analyses comparing outcomes of approved DMTs, and results from indirect comparisons provide helpful information to physicians that guide the choice of therapy [22-25]. Arguably, head-to-head randomized clinical trials comparing the efficacy and safety of DMTs are the gold standard for evidence-based health-care decision making. In the last year, results from two studies comparing the effectiveness of natalizumab and fingolimod were published. In randomized REVEAL study, natalizumab was shown to have a more rapid onset of efficacy, and to confer a greater benefit than fingolimod in reducing relapse rates and the number of gadolinium-enhancing lesions in patients with active RRMS [26]. Similarly, in the observational BEST-MS study, natalizumab was found to be more effective than fingolimod in reducing the number of relapses and MRI activity, thus yielding a higher rate of no evidence of disease activity after one year of treatment [27].

Taken together, these results suggest that natalizumab is highly effective, and that adherence to the recommended risk minimization measures can limit the number of AEs requiring treatment discontinuation, as well as the incidence of
SAEs, thus prolonging treatment duration and allowing for a higher proportion of patients to continue to derive a treatment benefit. Previous studies have suggested that longer duration of treatment with natalizumab may be associated with higher incidence of AEs, particularly progressive multifocal leukoencephalopathy (PML) [28]. However, in other analyses, the annual risk of PML started to decline after approximately 5 years of natalizumab therapy $[29,30]$. Further analyses are necessary to comprehensively inform on the risk of PML from natalizumab after long-term treatment.

The main limitations of the present study are those attributed to its observational design, including the absence of a control group, missing data, and patient selection bias. Another limitation pertains to the fact that during the two 6-month periods following approval of the original protocol and its first amendment, as well as during the 3-month period following its second amendment, enrolled patients could have had received any number of natalizumab infusions before enrollment. Therefore, under the perspective that for such patients to be continuing therapy they had gained benefit from treatment, there is the potential that enrollment of these patients has enhanced the effectiveness outcomes. Moreover, it should be noted that while for these patients, retrospective data collection of the effectiveness outcomes was utilized, no retrospective safety data collection took place, which could have artificially lowered the incidence of recorded SAEs. It is noted that the actual period between natalizumab initiation and enrollment in the study for the overall study population was 0.7 years. Furthermore, it should be taken into account that patients who were under relapse during EDSS evaluation were not excluded from the EDSS analysis, possibly leading to worse disability outcome estimation. Strengths of the study include its non-limiting eligibility criteria and enrollment of patients from 20 different sites, which strengthens generalizability of the findings 
to a population of patients with RRMS receiving natalizumab treatment under routine care conditions by hospitalbased physicians in Greece.

\section{Conclusions}

In conclusion, the present study provides evidence for the long-term effectiveness of natalizumab in patients with RRMS treated in routine care in Greece. Natalizumab reduced the clinical relapse rate during the first year of treatment, and the benefit was maintained throughout the course of the study. Moreover, natalizumab prevented disability accumulation, with a sustained effect during the study observation, and significantly decreased the baseline EDSS score over the first 3 years of treatment. The above favorable outcomes were accompanied by a predictable safety profile in terms of serious events, no opportunistic infections other than two cases of PML, a low treatment discontinuation rate due to AE occurrence, and a long drug survival. The results support the use of natalizumab in the routine clinical care for patients with highly active disease.

Acknowledgements The authors would like to thank Panagiota Karagianni from Qualitis Ltd. (CRO, Athens, Greece) for medical writing assistance funded by Genesis Pharma SA.

\section{Declarations}

Funding This study was funded by Genesis Pharma S.A.

Conflicts of interest The authors declare the following potential conflicts of interest with respect to the research, authorship, and/or publication of this article: Prof. Dardiotis received grants, consulting fees and travel support from Bayer, Novartis, Genesis Pharma, GenzymeSanofi, Merck-Serono, Roche and Teva. Dr. Evangelopoulou received grants and consulting fees from Roche, Merck, Biogen, Teva and Genzyme. George Karachalios, Alexopoulou Tania, Rania Gourgioti are employees of Genesis Pharma SA. No other potential conflict of interest relevant to this article was reported.

Data availability The authors declare that all relevant data supporting the findings of this study are available within the article.

Code availability Not applicable

Authors' contributions All authors contributed to the study conception and design, as well as the collection, analysis, and interpretation of data. All authors read and approved the final manuscript.

Ethics approval The study was approved by the ethics committees of all participating sites.

Consent to participate Informed consent was obtained from all individual participants included in the study.

Consent for publication Not applicable.
Open Access This article is licensed under a Creative Commons Attribution-NonCommercial 4.0 International License, which permits any non-commercial use, sharing, adaptation, distribution and reproduction in any medium or format, as long as you give appropriate credit to the original author(s) and the source, provide a link to the Creative Commons licence, and indicate if changes were made. The images or other third party material in this article are included in the article's Creative Commons licence, unless indicated otherwise in a credit line to the material. If material is not included in the article's Creative Commons licence and your intended use is not permitted by statutory regulation or exceeds the permitted use, you will need to obtain permission directly from the copyright holder. To view a copy of this licence, visit $\mathrm{http} / / /$ creativecommons.org/licenses/by-nc/4.0/.

\section{References}

1. Compston A, Coles A. Multiple sclerosis. Lancet. 2008;372(9648):1502-17. https://doi.org/10.1016/S01406736(08)61620-7.

2. Reich DS, Lucchinetti CF, Calabresi PA. Multiple sclerosis. N Engl J Med. 2018;378(2):169-80. https://doi.org/10.1056/NEJMr a1401483.

3. Lopes Pinheiro MA, Kooij G, Mizee MR, Kamermans A, Enzmann G, Lyck R, Schwaninger M, Engelhardt B, de Vries HE. Immune cell trafficking across the barriers of the central nervous system in multiple sclerosis and stroke. Biochim Biophys Acta. 2016;1862(3):461-71. https://doi.org/10.1016/j.bbadis.2015.10. 018.

4. Yednock TA, Cannon C, Fritz LC, Sanchez-Madrid F, Steinman L, Karin N. Prevention of experimental autoimmune encephalomyelitis by antibodies against alpha4beta1 integrin. Nature. 1992;356(6364):63-6. https://doi.org/10.1038/356063a0.

5. Baron JL, Madri JA, Ruddle NH, Hashim G, Janeway CA Jr. Surface expression of $\alpha 4$ integrin by CD4 T cells is required for their entry into brain parenchyma. J Exp Med. 1993;177(1):57-68. https://doi.org/10.1084/jem.177.1.57.

6. Ruegg C, Postigo AA, Sikorski EE, Butcher EC, Pytela R, Erle DJ. Role of integrin- $\alpha 4 \beta 7 / \alpha 4 \beta$ P in lymphocyte adherence to fibronectin and VCAM-1 and in homotypic cell clustering. J Cell Biol. 1992;117(1):179-89. https://doi.org/10.1083/jcb.117.1.179.

7. Bayless KJ, Meininger GA, Scholtz JM, Davis GE. Osteopontin is a ligand for the $\alpha 4 \beta 1$-integrin. J Cell Sci. 1998;111(Pt 9):1165-74.

8. Tysabri, summary of product characteristics. In: European Medicines Agency. 2020. https://www.ema.europa.eu/en/documents/ product-information/tysabri-epar-product-information_en.pdf. Accessed 8 Feb 2021.

9. Rudick RA, Sandrock A. Natalizumab: Alpha4-integrin antagonist selective adhesion molecule inhibitors for MS. Expert Rev Neurother. 2004;4(4):571-80. https://doi.org/10.1586/14737175.4.4. 571.

10. Ransohoff RM. Natalizumab for multiple sclerosis. N Engl J Med. 2007;356(25):2622-9. https://doi.org/10.1056/NEJMct071462.

11. Rudick R, Polman C, Clifford D, Miller D, Steinman L. Natalizumab: bench to bedside and beyond. JAMA Neurol. 2013;70(2):172-82. https://doi.org/10.1001/jamaneurol.2013.598.

12. Polman CH, O'Connor PW, Havrdova E, Hutchinson M, Kappos L, Miller DH, Phillips JT, Lublin FD, Giovannoni G, Wajgt A, Toal M, Lynn F, Panzara MA, Sandrock AW, AFFIRM Investigators. A randomized, placebo-controlled trial of natalizumab for relapsing multiple sclerosis. N Engl J Med. 2006;354(9):899-910. https://doi.org/10.1056/NEJMoa044397.

13. Karanasios P, Karachalios G, Gourgioti R, Alexopoulou A, Mastorodemos V. Patient and treatment characteristics and safety outcomes of patients with relapsing-remitting multiple sclerosis 
treated with natalizumab in Greece: results from the multicenter, 5 -year prospective observational study 'TOPICS greece'. Mult Scler J Exp Transl Clin. 2021. https://doi.org/10.1177/20552 173211035803.

14. Butzkueven H, Kappos L, Pellegrini F, Trojano M, Wiendl H, Patel RN, Zhang A, Hotermans C, Belachew S, TYSABRI Observational Program (TOP) Investigators. Efficacy and safety of natalizumab in multiple sclerosis: interim observational programme results. J Neurol Neurosurg Psychiatry. 2014;85(11):1190-7. https://doi.org/10.1136/jnnp-2013-306936.

15. Perumal J, Fox RJ, Balabanov R, Balcer LJ, Galetta S, Makh S, Santra S, Hotermans C, Lee L. Outcomes of natalizumab treatment within 3 years of relapsing-remitting multiple sclerosis diagnosis: a prespecified 2-year interim analysis of STRIVE. BMC Neurol. 2019;19(1):116. https://doi.org/10.1186/s12883-019-1337-z.

16. Trojano M, Butzkueven H, Kappos L, Wiendl H, Spelman T, Pellegrini F, Chen Y, Dong Q, Koendgen H, Belachew S, Tysabri Observational Program (TOP) Investigators. Natalizumab treatment shows low cumulative probabilities of confirmed disability worsening to EDSS milestones in the long-term setting. Mult Scler Relat Disord. 2018;24:11-9. https://doi.org/10.1016/j.msard. 2018.04.020.

17. Butzkueven H, Kappos L, Wiendl H, Trojano M, Spelman T, Chang I, Kasliwal R, Jaitly S, Campbell N, Ho PR, Licata S, Tysabri Observational Program (TOP) Investigators. Long-term safety and effectiveness of natalizumab treatment in clinical practice: 10 years of real-world data from the Tysabri Observational Program (TOP). J Neurol Neurosurg Psychiatry. 2020;91(6):660 8. https://doi.org/10.1136/jnnp-2019-322326.

18. Outteryck O, Ongagna JC, Brochet B, Rumbach L, Lebrun-Frenay C, Debouverie M, Zéphir H, Ouallet JC, Berger E, Cohen M, Pittion S, Laplaud D, Wiertlewski S, Cabre P, Pelletier J, Rico A, Defer G, Derache N, Camu W, Thouvenot E, Moreau T, Fromont A, Tourbah A, Labauge P, Castelnovo G, Clavelou P, Casez O, Hautecoeur P, Papeix C, Lubetzki C, Fontaine B, Couturier N, Bohossian N, Clanet M, Vermersch P, de Sèze J, Brassat D, BIONAT Network, CFSEP. A prospective observational post-marketing study of natalizumab-treated multiple sclerosis patients: clinical, radiological and biological features and adverse events. The BIONAT cohort. Eur J Neurol. 2014;21(1):40-8. https://doi. org/10.1111/ene.12204.

19. Guger M, Enzinger C, Leutmezer F, Kraus J, Kalcher S, Kvas E, Berger T. Real-life clinical use of natalizumab and fingolimod in Austria. Acta Neurol Scand. 2018;137(2):181-7. https://doi.org/ 10.1111/ane.12864

20. Lanzillo R, Carotenuto A, Moccia M, Saccà F, Russo CV, Massarelli M, De Rosa A, Brescia MV. A longitudinal real-life comparison study of natalizumab and fingolimod. Acta Neurol Scand. 2017;136(3):217-22. https://doi.org/10.1111/ane.12718.

21. Dekker I, Leurs CE, Hagens MHJ, van Kempen ZLE, Kleerekooper I, Lissenberg-Witte BI, Barkhof F, Uitdehaag BMJ, Balk LJ, Wattjes MP, Killestein J. Long-term disease activity and disability progression in relapsing-remitting multiple sclerosis patients on natalizumab. Mult Scler Relat Disord. 2019;33:82-7. https://doi. org/10.1016/j.msard.2019.05.017.
22. Tsivgoulis G, Katsanos AH, Mavridis D, Grigoriadis N, Dardiotis E, Heliopoulos I, Papathanasopoulos P, Karapanayiotides T, Kilidireas C, Hadjigeorgiou GM, Voumvourakis K. The efficacy of natalizumab versus fingolimod for patients with relapsing-remitting multiple sclerosis: a systematic review, indirect evidence from randomized Placebo-controlled trials and meta-analysis of observational head-to-head trials. PLoS ONE. 2016;11(9):e0163296. https://doi.org/10.1371/journal.pone.0163296.

23. Lorscheider J, Benkert P, Lienert C, Hänni P, Derfuss T, Kuhle J, Kappos L, Yaldizli Ö. Comparative analysis of natalizumab versus fingolimod as second-line treatment in relapsing-remitting multiple sclerosis. Mult Scler. 2018;24(6):777-85. https://doi.org/ $10.1177 / 1352458518768433$.

24. Barbin L, Rousseau C, Jousset N, Casey R, Debouverie M, Vukusic S, De Sèze J, Brassat D, Wiertlewski S, Brochet B, Pelletier J, Vermersch P, Edan G, Lebrun-Frenay C, Clavelou P, Thouvenot E, Camdessanché JP, Tourbah A, Stankoff B, Al Khedr A, Cabre P, Papeix C, Berger E, Heinzlef O, Debroucker T, Moreau T, Gout O, Bourre B, Créange A, Labauge P, Magy L, Defer G, Foucher Y, Laplaud DA. Comparative efficacy of fingolimod vs natalizumab: a French multicenter observational study. Neurology. 2016;86(8):771-8. https://doi.org/10.1212/WNL.0000000000 002395.

25. Meca-Lallana J, Ayuso T, Martínez-Yelamos S, Durán C, Contreras Martín Y, Herrera Navarro N, Pérez Sempere A, ÁlvarezCermeño JC, Millán Pascual J, Meca-Lallana V, Romero Sevilla R, Ricart J. Effectiveness of fingolimod versus natalizumab as second-line therapy for relapsing-remitting multiple sclerosis in Spain: second-line GATE Study. Eur Neurol. 2020;83(1):25-33. https://doi.org/10.1159/000505778.

26. Butzkueven H, Licata S, Jeffery D, Arnold DL, Filippi M, Geurts JJ, Santra S, Campbell N, Ho PR. Natalizumab versus fingolimod for patients with active relapsing-remitting multiple sclerosis: results from REVEAL, a prospective, randomised head-to-head study. BMJ Open. 2020;10(10):e038861. https://doi.org/10.1136/ bmjopen-2020-038861.

27. Cohen M, Mondot L, Bucciarelli F, Pignolet B, Laplaud DA, Wiertlewski S, Brochet B, Ruet A, Defer G, Derache N, Vermersch P, Zephir H, Debouverie M, Mathey G, Berger E, Cappé C, Labauge P, Carra C, De Seze J, Bigaut K, Brassat D, LebrunFrenay C. BEST-MS: a prospective head-to-head comparative study of natalizumab and fingolimod in active relapsing MS. Mult Scler. 2020;2020. https://doi.org/10.1177/1352458520969145.

28. Foley J, Carrillo-Infante C, Smith J, Evans K, Ho PR, Lee L, Kasliwal R, Stangel M, Vermersch P, Hutchinson M, Marinelli F, Smirnakis K. The 5-year Tysabri global observational program in safety (TYGRIS) study confirms the long-term safety profile of natalizumab treatment in multiple sclerosis. Mult Scler Relat Disord. 2019;39:101863. https://doi.org/10.1016/j.msard.2019. 101863.

29. Ho PR, Campbell N, Chang I. The risk of PML from natalizumab-authors' reply. Lancet Neurol. 2019;18(3):230-1. https:// doi.org/10.1016/S1474-4422(19)30022-5.

30. Tugemann B, Berger JR. Improving risk-stratification of natalizumab-associated PML. Ann Clin Transl Neurol. 2021;8(3):696703. https://doi.org/10.1002/acn3.51130. 